\title{
Aberrant expression and potential therapeutic target of lysophosphatidic acid receptor 3 in triple-negative breast cancers
}

\author{
Kai Sun $\cdot$ Hui Cai $\cdot$ Xiaoyi Duan $\cdot$ Ya Yang $\cdot$ \\ Min Li $\cdot$ Jingkun Qu $\cdot$ Xu Zhang $\cdot$ Jiansheng Wang
}

Received: 9 June 2014/ Accepted: 28 July 2014/Published online: 11 September 2014

(c) The Author(s) 2014. This article is published with open access at Springerlink.com

\begin{abstract}
Triple receptor-negative breast cancers (TNBCs) generally have poor prognoses because of the loss of therapeutic targets. As lysophosphatidic acid (LPA) receptor signaling has been shown to affect breast cancer initiation and progression, we try to evaluate the potential roles of LPA receptors in TNBCs. We examined mRNA and protein expressions of LPA receptors 1-3, using quantitative real-time PCR and immunohistochemical analyses in normal $(n=37)$, benign disease $(n=55)$, and breast cancer tissues $(n=82)$. Carcinomas expressed higher levels of $\mathrm{LPA}_{2}$ and $\mathrm{LPA}_{3}$ mRNAs $(0.17 \pm 0.070$ and $0.05 \pm 0.023$, respectively) than did normal breast tissue $(0.13 \pm 0.072$ and $0.02 \pm 0.002$, respectively). Enhanced immunohistochemical staining for $\mathrm{LPA}_{2}$ and $\mathrm{LPA}_{3}$ protein was also consistently observed in carcinomas. The $\mathrm{LPA}_{3}$ overexpression was associated with lymph
\end{abstract}

Kai Sun and Hui Cai have contributed equally to this work.

K. Sun · Y. Yang $\cdot$ M. Li · J. Qu · J. Wang $(\varangle)$

The Second Department of Thoracic Surgery, The First

Affiliated Hospital of Xi' an Jiaotong University, 277 West Yanta

Road, Xi' an 710061, Shaanxi, China

e-mail: wangjsh@mail.xjtu.edu.cn; wangjshxjtu@gmail.com

H. Cai

Department of Anesthesia, The First Affiliated Hospital of Xi' an Jiaotong University, Xi'an, Shaanxi, China

X. Duan $(\square)$

Department of Nuclear Medicine, The First Affiliated Hospital of Xi' an Jiaotong University, 277 West Yanta Road,

Xi'an 710061, Shaanxi, China

e-mail: duanxy@mail.xjtu.edu.cn

X. Zhang

Department of Pathology, Lanzhou University Medical School, Lanzhou, Gansu, China node metastases, and absence of estrogen receptor, progesterone receptors, and human epidermal growth factor receptor 2 expression. TNBC tissues and cell lines showed the highest $\mathrm{LPA}_{3}$ expression compared with luminal-type $A$ and $B$ breast cancers. Suppression of $\mathrm{LPA}_{3}$ by shRNA did not influence cell growth in breast cancer cells. However, the migration and invasion of TNBC cells were significantly inhibited by $\mathrm{LPA}_{3}$-shRNA or inhibitor, which had no or less effect on normal and non-TNBC breast cells. In conclusion, our data indicated that the expression of LPA receptor 3 was increased in human TNBCs and is associated with tumor metastatic ability, and this implies that $\mathrm{LPA}_{3}$ is a potential therapeutic target for the treatment of TNBCs.

Keywords Lysophosphatidic acid receptor - Triple receptor-negative breast cancer - Aberrant expression . Therapeutic target

\section{Introduction}

Breast cancer is the most commonly diagnosed cancer, and the leading cause of cancer-related deaths, in women worldwide [1]. Cases are usually classified by their expression of estrogen receptors (ER) progesterone receptors (PR), and human epidermal growth factor-2 receptors (HER2), which together predict treatment response and prognosis [2]. Although hormone receptor $(\mathrm{HR})^{+}$breast cancers have many effective treatment options, fewer targeted therapies are available for triple receptor-negative breast cancers (TNBCs). Currently, some progress has been made in classifying TNBCs into several distinct subtypes using gene expression profiling analyses, and some kinases and agents were identified as potential druggable targets [3, 
4], but the therapeutic implications are yet to be elucidated [5]. Thus, characterization of novel molecular biomarkers is critically required for the treatment of TNBCs.

Lysophosphatidic acid (LPA) receptors are specific G protein-coupled receptors binding with LPA, which mediates a variety of biological processes, such as cell proliferation, migration, invasion and differentiation [6]. At least six LPA receptors $\left(\mathrm{LPA}_{1-6}\right)$ are currently identified, and their emerging roles in tumorigenesis have been demonstrated both in vitro and in vivo [7]. In breast tissue, $\mathrm{LPA}_{1}$ and $\mathrm{LPA}_{2}$ are broadly expressed in either normal or abnormal mammary epithelial cells, whereas expression levels of $\mathrm{LPA}_{3-6}$ are more restricted or undetectable, which may account for the various biological effects of LPA [810]. Overexpression of $\mathrm{LPA}_{1}$ and $\mathrm{LPA}_{2}$ was readily observed in breast cancers with redundant mediation functions in multiple endogenous LPA responses, including cancer cell growth, metastasis, angiogenesis, and chemoresistance [8, 11-13]. In contrast, less is known about the role of $\mathrm{LPA}_{3}$ in breast cancer initiation and progression. Previous published data showed that $\mathrm{LPA}_{3}$ was higher expression in poorly differentiated breast cancers than well-differentiated tumors $[14,15]$, which suggests that $\mathrm{LPA}_{3}$ contributes to breast cancer progression.

Although the LPA receptors have been shown to affect breast cancer initiation and progression, the exact expression patterns and functions in TNBCs have not been fully examined. In the present study, we characterized the expression of $\mathrm{LPA}_{1-3}$ in human normal, benign, and malignant breast tissues and cell lines, and analyzed correlations with clinical and pathological findings to highlight the possible roles of LPA receptors in the development and treatment of TNBCs.

\section{Materials and methods}

\section{Patients}

Specimens of normal breast $(n=37)$, mammaries with benign disease $(n=55)$, and breast cancer $(n=82)$ were collected from the First Affiliated Hospital of Xi'an Jiaotong University. This study was approved by the IRB of Xi' an Jiaotong University School of Medicine. All tissues were pathologically examined. Written informed consent forms were obtained from all subjects, and all clinical investigation had been conducted according to the principles expressed in the Declaration of Helsinki.

RNA isolation and quantitative real-time PCR

Tissues or cells were lysed in the Qiagen RLT lysis buffer (Qiagen, USA). RNA was extracted with an RNeasy mini kit (Qiagen, USA) and reverse transcribed by M-MLV reverse transcriptase (Invitrogen, USA). Quantitative realtime PCR was performed on a Bio-Rad iQ5 Real-Time PCR Detection System (Bio-Rad Laboratories, USA) with a SYBR Green I Master Mix (TAKARA, Japan). PCRs were performed in triplicate, and the relative gene expression was calculated against GAPDH. Primer pairs used in this study were as follows: GAPDH: F, 5'-GAAGGTGAAGGTCGGAGT-3'/R, 5'-GAAGATGGTGATG GGATTTC-3'; LPA $_{1}$ : F, 5'-AATCGAGAGGCACATTAC GG-3'/R, 5'-GTTGAAAATGGCCCAGAAGA-3'; LPA $_{2}$ : F, 5'-TTGTCTTCCTGCTCATGGTG-3'/R, 5'-TCAGCAT CTCGGCAAGAGTA-3'; LPA $_{3}$ : F, 5'-TGCTCATTTTGC TTGTCTGG-3'/R, 5'-GCCATACATGTCCTCGTCCT-3'

Immunohistochemistry (IHC) analysis

Formalin-fixed paraffin-embedded sections ( $5 \mu \mathrm{m}$ thick) of the normal breast, breast with benign diseases, and breast cancers were analyzed by IHC with the primary $\mathrm{LPA}_{1-3}$ antibody (1:100) and a biotin-conjugated secondary antibody. For IHC quantification, the sections were analyzed using Nikon TE2000-s microscope (Melville, USA). Four randomly selected areas were photographed at $40 \times$ magnification using a QimageRetiga 2000R camera (Surrey, Canada). The integral optical density (IOD) of immunoreactivity was calculated using the Image-Pro Plus image analysis software (Media Cybernetics, USA).

\section{Cell lines and culture}

The MCF-10A and MCF-7 cells were obtained from Sagene Inc., (Guangzhou, China), and the MCF-12A, T47D, MDA-MB-231, and MDA-MB-157 cells were obtained from ATCC (Manassas, USA). All cell lines were maintained in a humidified atmosphere at $37{ }^{\circ} \mathrm{C}$ with $5 \% \mathrm{CO}_{2}$. MCF-10A, MCF-12A, and MCF-7 cells were cultured in DMEM with glutamine, $10 \%$ FBS (Gibco, USA), and $100 \mu \mathrm{g} / \mathrm{mL}$ penicillin/streptomycin (P/S). T47D were cultured in RPMI1640 with glutamine, $10 \%$ FBS (Gibco), and $100 \mu \mathrm{g} / \mathrm{mL}$ P/S. MDA-MB-231 and MDA-MB-157 cultured in Leibovitz's L-15 Medium (ATCC, USA) with $10 \%$ FBS (Gibco) and $100 \mu \mathrm{g} / \mathrm{mL} \mathrm{P} / \mathrm{S}$.

\section{Western blot analyses}

Western blot analyses were conducted using standard procedures, and proteins were detected using primary antibodies and fluorescent secondary antibodies (IRDye800CW-conjugated or IRDye680-conjugated anti-species IgG, Li-Cor Biosciences, Lincoln, NE, USA). The fluorescent signals were captured on an Odyssey Infrared Imaging System (Li-Cor Biosciences) with both 700- and 
800-nm channels. Boxes were manually placed around each band of interest, and the software returned nearinfrared fluorescent values of raw intensity with background subtraction (Odyssey 3.0 analytical software, LiCor Biosciences).

\section{shRNA transfection}

Six-well plates were seeded with $5 \times 10^{4}$ cells/well in $2 \mathrm{~mL}$ media $24 \mathrm{~h}$ before transfection; cells were $80-90 \%$ confluent at transfection. Cells were transfected with $\mathrm{LPA}_{3}$ shRNA (100 pmol/well, Santa Cruz Biotechnology, USA) using Lipofectamine 2000 Reagent (Life Technologies, USA) according to the manufacturer's instruction. After $48 \mathrm{~h}$ of transfection, cells were selected using puromycin for 2 weeks. Stable transductants were pooled.

\section{MTT assays}

Cells were seeded at a density of $5 \times 10^{3}$ cells/well in 96-well plates at a final volume of $180 \mu \mathrm{L}$ in incubation, at $37{ }^{\circ} \mathrm{C}$, with $5 \% \mathrm{CO}_{2}$. After different time incubation, $20 \mu \mathrm{L}$ of $5 \mathrm{mg} / \mathrm{mL}$ solution of MTT (Sigma, MO, USA) in PBS was added to each well, and plates were then incubated for $4 \mathrm{~h}$ at $37^{\circ} \mathrm{C}$. Reaction crystals were then solubilized in $100 \%$ dimethylsulfoxide (Sigma) $20 \mu \mathrm{L} /$ well and shaken for $15 \mathrm{~min}$. Absorbance of each well was measured on a multidetection microplate reader (BMG LABTECH, USA) at a wavelength of $570 \mathrm{~nm}$.

\section{Cell migration and invasion Assays}

Migration and invasion assays were conducted using transwell plates with $8-\mu \mathrm{m}$ pore size membranes (Corning Inc., USA) as described previously [16]. After incubation for $4 \mathrm{~h}$ (for migration assays) or $24 \mathrm{~h}$ (for invasion assays), cells remaining in the upper side of the filter were removed with cotton swabs. The cells attached on the lower surface were fixed and stained using crystal violet and washed with water. Cells were counted with five high power fields per membrane, and results were presented as the mean number of cells migrated per field per membrane. All experiments were conducted in triplicate.

\section{Statistical analyses}

Continuous variables were summarized as means with standard deviations (SD) across the healthy control, benign disease, and cancer groups. One-way ANOVA was used to test the overall difference, and Student's $t$ test was used to test the pairwise difference across disease statuses. Correlation between two different groups was tested by Pearson's correlation coefficient. $P<0.05$ was considered significant. All analyses were performed using SPSS software version 19.0 (IBM, USA).

\section{Results}

Expression patterns of $\mathrm{LPA}_{1-3}$ in breast tissues

We evaluated mRNA expression of $\mathrm{LPA}_{1-3}$ in normal, benign, and malignant breast epithelium; mRNA levels were quantified against $G A P D H$. As shown in Fig. 1a, breast tissues predominantly expressed $\mathrm{LPA}_{1}$ and $\mathrm{LPA}_{2}$, whereas $\mathrm{LPA}_{3}$ expression was weakly but detectable in all specimens. Similar levels of $\mathrm{LPA}_{1}$ mRNA were detected in normal, benign, and carcinoma tissues $(0.11 \pm 0.058$ vs. $0.13 \pm 0.044$ vs. $0.13 \pm 0.034, \quad P=0.789 ; \quad$ Fig. 1b) However, $\mathrm{LPA}_{2}$ mRNA levels in breast cancers were significantly higher than that in normal tissue $(0.17 \pm 0.070$ vs. $0.13 \pm 0.072, P=0.0002$; Fig. 1c). Although low levels of $\mathrm{LPA}_{3}$ were observed in all breast tissues, the cancer tissues exhibited a greater expression of $\mathrm{LPA}_{3}$ than did normal $(0.05 \pm 0.023$ vs. $0.02 \pm 0.002, P<0.001)$ or benign-disease tissues $(0.05 \pm 0.023$ vs. $0.03 \pm 0.002$, $P<0.001$ ) (Fig. 1d). Notably, $\mathrm{LPA}_{3}$ expression was also
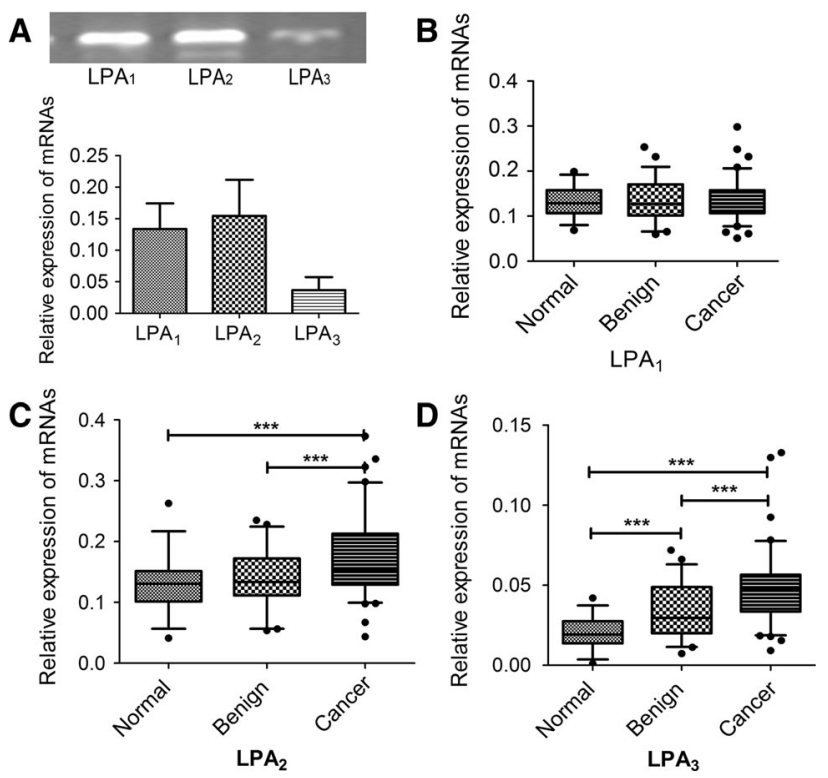

Fig. 1 mRNA expression of LPA receptor 1-3 in breast tissues. a To determine whether the samples expressed LPA receptors, quantitative real-time PCR was performed by $\mathrm{LPA}_{1}, \mathrm{LPA}_{2}$, and $\mathrm{LPA}_{3}$ primers. The relative gene expression was calculated against GAPDH. b The relative $\mathrm{LPA}_{1}$ mRNA expression in normal breast epithelium, mammary with benign disease, and malignant tissues. c The relative $\mathrm{LPA}_{2}$ mRNA expression in normal breast epithelium, mammary with benign disease, and malignant tissues. d The relative $\mathrm{LPA}_{3}$ mRNA expression in normal breast epithelium, mammary with benign disease, and malignant tissues. $* * * P<0.001$ 
greater in benign-disease tissue than in normal tissue $(0.03 \pm 0.002$ vs. $0.02 \pm 0.002, P=0.009$; Fig. 1 d) .

We also immunohistochemically evaluated expression of LPA receptor proteins in the same specimens (Fig. 2a). $\mathrm{LPA}_{1-3}$ was detectable in the cell membrane and cytoplasm in most specimens (113/119 of $\mathrm{LPA}_{1}, 116 / 119$ of $\mathrm{LPA}_{2}$, and $110 / 119$ of $\left.\mathrm{LPA}_{3}\right)$. As with the mRNA expression, enhanced staining for $\mathrm{LPA}_{2}$ and $\mathrm{LPA}_{3}$ protein was clearly detected in carcinomas in comparison with normal epithelium or benign-disease tissues (Fig. 2c, d), whereas $\mathrm{LPA}_{1}$ expression did not differ significantly between different groups (Fig. 2b). Protein immunoreactivity significant correlated with relative mRNA expression $(r=0.592, P<0.001)$.

Relationship between $\mathrm{LPA}_{1-3}$ mRNA expression and clinical parameters in breast cancer patients

Relationships between $\mathrm{LPA}_{1-3}$ mRNA expression and clinical or pathological findings in 82 cases are presented in Table $1 . \mathrm{LPA}_{1}$ expression did not correlate with any clinical parameters. Higher expression of $\mathrm{LPA}_{2}$ was seen in postmenopausal patients $(P<0.05)$. The higher-stage tumors tended to express less $\mathrm{LPA}_{2}$, but not significantly

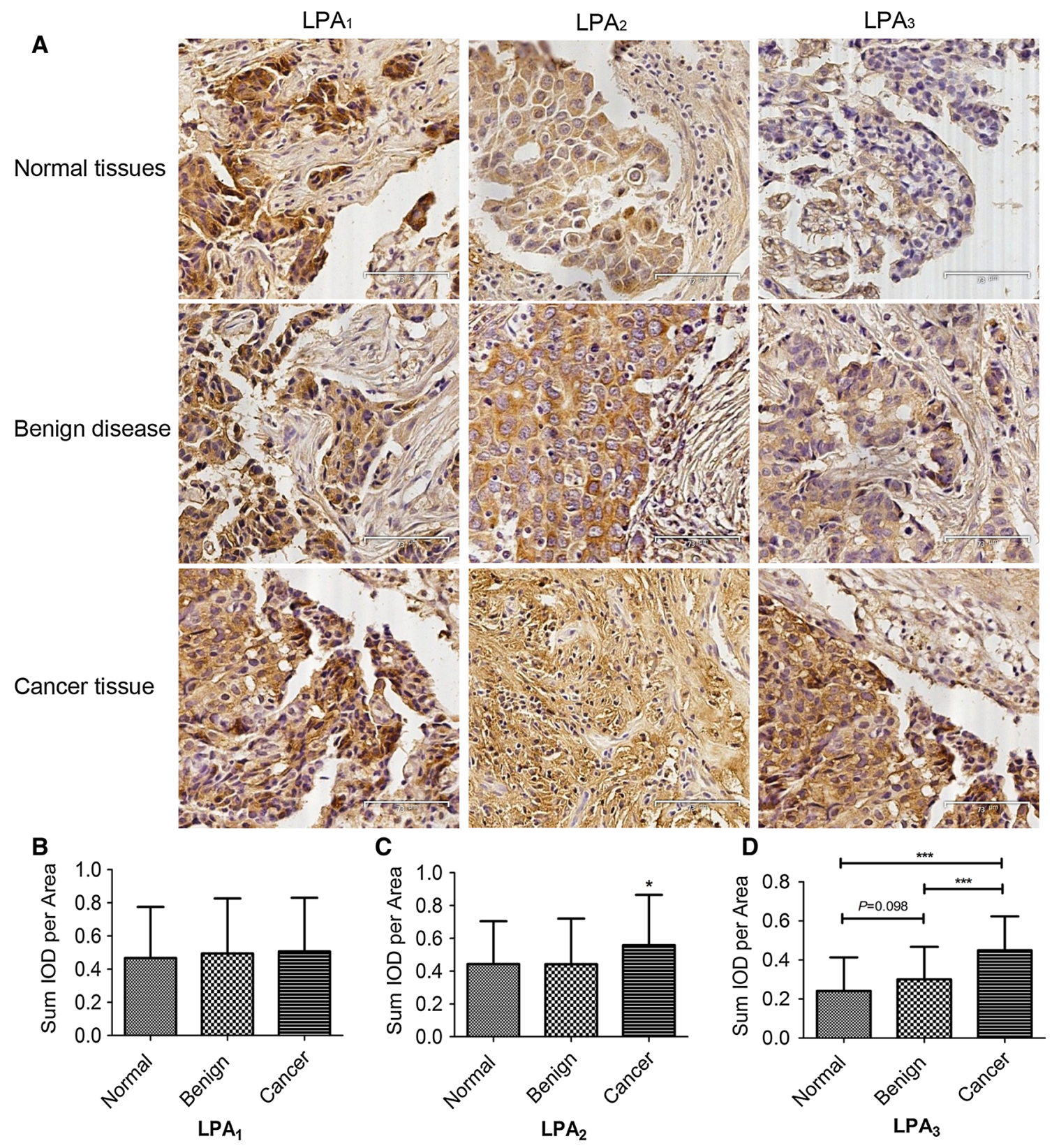

Fig. 2 Protein levels of LPA receptor 1-3 in different breast tissues. a Immunostains for LPA receptor 1-3 in normal, benign disease and malignant breast tissue. b-d Quantification of immunostains for LPA receptor 1-3 by IOD analysis. $* P<0.05 ; * * * P<0.001$ 
Table 1 Relationship between LPA receptors expression and clinical parameters of breast cancer
$* P<0.05 ; * * P<0.01$;

$* * * P<0.001$

\begin{tabular}{|c|c|c|c|c|}
\hline Clinical parameters & $N(\%)$ & $\mathrm{LPA}_{1}$ & $\mathrm{LPA}_{2}$ & $\mathrm{LPA}_{3}$ \\
\hline \multicolumn{5}{|l|}{ Age (years) } \\
\hline$<50$ & $41(50.0)$ & $0.134 \pm 0.039$ & $0.173 \pm 0.064$ & $0.043 \pm 0.020$ \\
\hline$\geq 50$ & $41(50.0)$ & $0.133 \pm 0.045$ & $0.172 \pm 0.065$ & $0.051 \pm 0.022$ \\
\hline \multicolumn{5}{|l|}{ Stage } \\
\hline I & $14(17.0)$ & $0.134 \pm 0.047$ & $0.184 \pm 0.059$ & $0.047 \pm 0.030$ \\
\hline II & $13(15.9)$ & $0.141 \pm 0.042$ & $0.178 \pm 0.058$ & $0.048 \pm 0.014$ \\
\hline III & $20(24.4)$ & $0.127 \pm 0.028$ & $0.173 \pm 0.069$ & $0.041 \pm 0.016$ \\
\hline IV & $35(42.7)$ & $0.134 \pm 0.047$ & $0.167 \pm 0.066$ & $0.050 \pm 0.022$ \\
\hline \multicolumn{5}{|l|}{ Grade } \\
\hline 1 & $29(35.4)$ & $0.134 \pm 0.036$ & $0.177 \pm 0.060$ & $0.040 \pm 0.016$ \\
\hline 2 & $37(45.1)$ & $0.136 \pm 0.048$ & $0.164 \pm 0.071$ & $0.050 \pm 0.026$ \\
\hline 3 & $16(19.5)$ & $0.125 \pm 0.048$ & $0.184 \pm 0.070$ & $0.051 \pm 0.017$ \\
\hline \multicolumn{5}{|l|}{ Tumor size } \\
\hline$\leq 2.0 \mathrm{~cm}$ & $30(36.6)$ & $0.124 \pm 0.036$ & $0.173 \pm 0.063$ & $0.049 \pm 0.023$ \\
\hline $2.0-5.0 \mathrm{~cm}$ & $36(43.9)$ & $0.143 \pm 0.046$ & $0.177 \pm 0.068$ & $0.049 \pm 0.020$ \\
\hline$>5.0 \mathrm{~cm}$ & $16(19.5)$ & $0.130 \pm 0.039$ & $0.163 \pm 0.058$ & $0.037 \pm 0.019$ \\
\hline \multicolumn{5}{|l|}{ Menopausal status } \\
\hline Premenopausal & $32(39.0)$ & $0.133 \pm 0.043$ & $0.154 \pm 0.046^{*}$ & $0.043 \pm 0.022$ \\
\hline Postmenopausal & $50(61.0)$ & $0.134 \pm 0.041$ & $0.185 \pm 0.071$ & $0.049 \pm 0.021$ \\
\hline \multicolumn{5}{|l|}{ ER status } \\
\hline Negative & $37(45.1)$ & $0.133 \pm 0.045$ & $0.169 \pm 0.060$ & $0.057 \pm 0.023 * * *$ \\
\hline Positive & 45 (54.9) & $0.134 \pm 0.039$ & $0.176 \pm 0.067$ & $0.038 \pm 0.015$ \\
\hline \multicolumn{5}{|l|}{ PR status } \\
\hline Negative & $43(52.4)$ & $0.132 \pm 0.044$ & $0.180 \pm 0.072$ & $0.055 \pm 0.023 * * *$ \\
\hline Positive & $39(47.6)$ & $0.135 \pm 0.040$ & $0.166 \pm 0.054$ & $0.037 \pm 0.014$ \\
\hline \multicolumn{5}{|l|}{ Her2 status } \\
\hline Negative & $55(67.1)$ & $0.135 \pm 0.043$ & $0.164 \pm 0.055$ & $0.051 \pm 0.023 * *$ \\
\hline Positive & $27(32.9)$ & $0.131 \pm 0.041$ & $0.191 \pm 0.077$ & $0.038 \pm 0.013$ \\
\hline \multicolumn{5}{|l|}{ Nodal metastasis } \\
\hline Negative & $37(45.1)$ & $0.132 \pm 0.042$ & $0.177 \pm 0.064$ & $0.034 \pm 0.013 * * *$ \\
\hline Positive & $45(54.9)$ & $0.135 \pm 0.042$ & $0.170 \pm 0.064$ & $0.057 \pm 0.021$ \\
\hline
\end{tabular}

$(P=0.095)$. The expression of $\mathrm{LPA}_{3}$ was associated with hormonal receptor status and lymph node metastases. $\mathrm{ER}^{-}$, $\mathrm{PR}^{-}$, or Her ${ }^{-}$tumors were more likely to express excess $\mathrm{LPA}_{3}$ than positive ones. Moreover, patients with lymph node metastases presented with higher $\mathrm{LPA}_{3}$ expression than patients without metastases $(P<0.05)$.

Higher expression of $\mathrm{LPA}_{3}$ in TNBC tissues and cell lines

As $\mathrm{LPA}_{3}$ expression in carcinomas strongly correlated with HR status, we subsequently analyzed the distributions of $\mathrm{LPA}_{3}$ among different tumor immunophenotypes. Breast cancer patients were classified as luminal A, luminal B, and TNBC, based on their expression of ER, PR, Her2, and ki67 [17]. $\mathrm{LPA}_{3}$ expression differed significantly among tumors with different immunophenotypes $(P<0.001$; Fig. 3a). The highest $\mathrm{LPA}_{3}$ protein level was demonstrated in the TNBCs whereas similar expressions were found between luminal A and luminal B carcinomas.

To confirm the expression profiles of $\mathrm{LPA}_{3}$ in TNBCs, we further detected the mRNA and protein levels of $\mathrm{LPA}_{3}$ in normal mammary epithelial cells and breast cancer cell lines with different molecular phenotypes. As expected, breast cancer cell lines (MCF-7, T47D, MDA-MB-231, and MDA-MB-157) expressed more $\mathrm{LPA}_{3}$ than normal immortal cells (MCF-10A and MCF-12A), and the highest expression of $\mathrm{LPA}_{3}$ was detected in the TNBC cells (MDA-MB-231 and MDA-MB-157) (Fig. 3b, c).

Inhibition of $\mathrm{LPA}_{3}$ by shRNA decreased migration and invasion of TNBC cells

To further analyze the role of $\mathrm{LPA}_{3}$ in breast tumorigenesis, we conducted cell proliferation, migration, and invasion assays of $\mathrm{LPA}_{3}$ - and control-shRNA-transfected breast 
Fig. 3 High expression of $\mathrm{LPA}_{3}$ in TNBCs. a The relative LPA3 mRNA expression in breast cancer tissues from luminal A, luminal B and TNBC patients. b The relative $\mathrm{LPA}_{3}$ mRNA expression in six different breast cell lines was determined by quantitative realtime PCR. The results are presented as the mean $\pm \mathrm{SD}$ against GAPDH obtained in three independent experiments. c Western blots were used to detect protein levels of $\mathrm{LPA}_{3}$ in six breast cell lines.

Quantification of protein was presented as the mean $\pm \mathrm{SD}$ of fluorescent values obtained in three independent experiments. ${ }^{\dagger}$ Compared to normal mammary cells, $P<0.05 ;{ }^{*}$ compared to normal mammary cells or nonTNBC cells, $P<0.001$
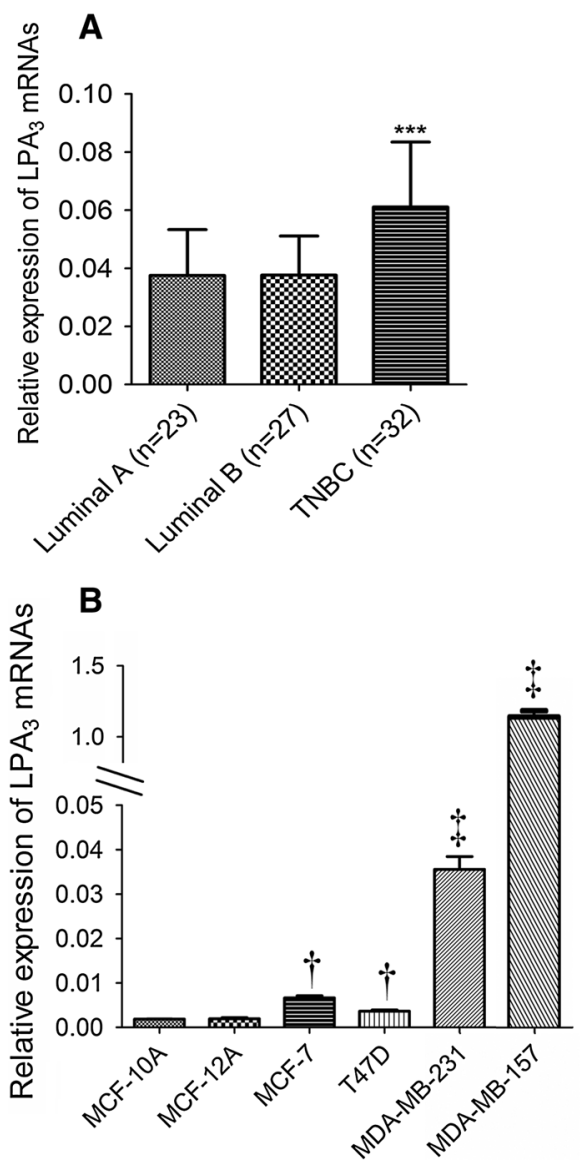
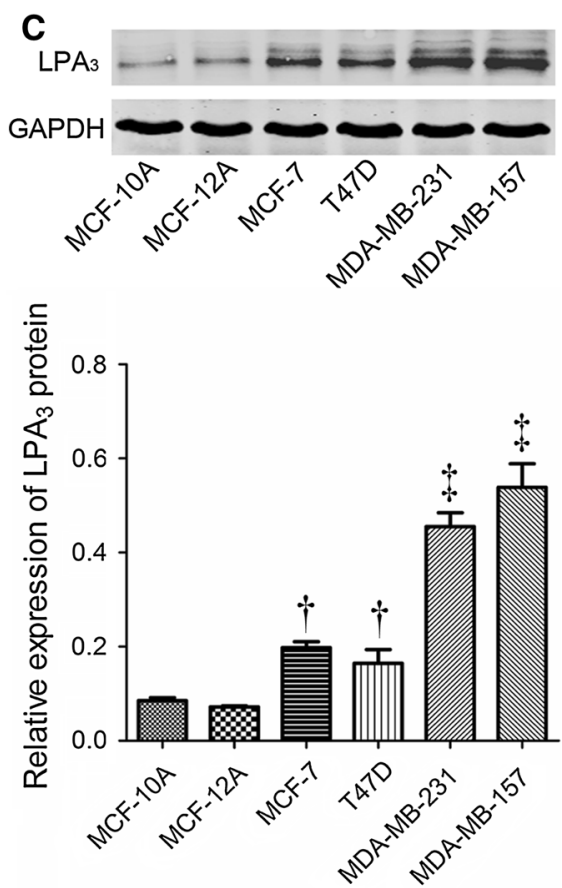

epithelial cells, including normal immortal cells MCF-10A, luminal cells MCF-7, and TNBC cells MDA-MB-231. $\mathrm{LPA}_{3}$ was effectively down-regulated by shRNA in all three cell lines (Fig. 4a). Cell proliferation tested by MTT showed that suppression of $\mathrm{LPA}_{3}$ did not influence cell growth in all three cell lines (Fig. 4b). However, cells with $\mathrm{LPA}_{3}$-shRNA migrated significantly less than controls in MDA-MB-231 cells (Fig. 4c). Although LPA 3 -shRNA also reduced migration of $\mathrm{MCF}-7$ cells, the inhibitory capacity was weaker than in MDA-MB-231 cells (Fig. 4c). We also assessed the effect of $\mathrm{LPA}_{3}$ knockdown on cellular invasion and revealed $\mathrm{LPA}_{3}$ loss significantly decreased invasion of MDA-MB-231 cells, but had less or no effect on MCF-10A and MCF-7 cells (Fig. 4d).

Ki16425 dose-dependently suppressed migration and invasion of TNBC cells

We used Ki16425, an antagonist for $\mathrm{LPA}_{1}$ and $\mathrm{LPA}_{3}$, to confirm the critical roles of $\mathrm{LPA}_{3}$ in TNBC cells. We first showed that pre-treating MDA-MB-231 cells with Ki16425 did not influence cell viability (Fig. 5a). We then evaluated the effects of Ki16425 on migration and invasion of TNBC cells, using transwell assays. As shown in Fig. 5b, c,
Ki16425 suppressed migration and invasion of MDA-MB231 cells in a dose-dependent manner.

\section{Discussion}

LPA receptors are expressed by normal mammary epithelial cells, with aberrant expression occurring during breast cancer initiation and progression [18]. In the present study, we found abnormal expression of LPA receptors in mammary carcinomas, and that $\mathrm{LPA}_{2}$ and $\mathrm{LPA}_{3}$ expression was enhanced in breast cancer compared with normal breast and benign-disease tissues, although the expression level of LPA $_{1}$ was not significantly different between each subgroup. Particularly, we also showed significantly increased $\mathrm{LPA}_{3}$ expression in the TNBCs compared with other immunophenotype tumors. Subsequently, function analysis revealed that inhibition of $\mathrm{LPA}_{3}$ by shRNA or antagonist dramatically suppressed the migration and invasion ability of TNBC cells, but had no or less effect on normal or luminal-type cancer cells, which suggests a role for $\mathrm{LPA}_{3}$ in the pathophysiology of TNBCs.

Expression and function of $\mathrm{LPA}_{1}$ in the breast cancer have been studied extensively. Overexpression of $\mathrm{LPA}_{1}$ is 
Fig. 4 Inhibition of $\mathrm{LPA}_{3}$ decreased migration and invasion of TNBC cells. a Expression of $\mathrm{LPA}_{3}$ was decreased by shRNA. MCF-

231 cells were transfected with control and $\mathrm{LPA}_{3}$ shRNA.

Forty-eight hours later, cell lysates were analyzed by Western blots with anti-LPA antibody. b The effect of $\mathrm{LPA}_{3}$ on breast cancer cells growth, as measured using the MTT assay. The results are presented as the mean \pm SD of fold increased to initiation obtained in 3 independent experiments. c, d Cell transwell assays were conducted to investigate the role of $\mathrm{LPA}_{3}$ on breast cancer cells migration (c) and invasion (d). The results are presented as the mean \pm SD of cell number obtained in three independent experiments. $* * P<0.01$; $* * * P<0.001$ 10A, MCF-7, and MDA-MB-
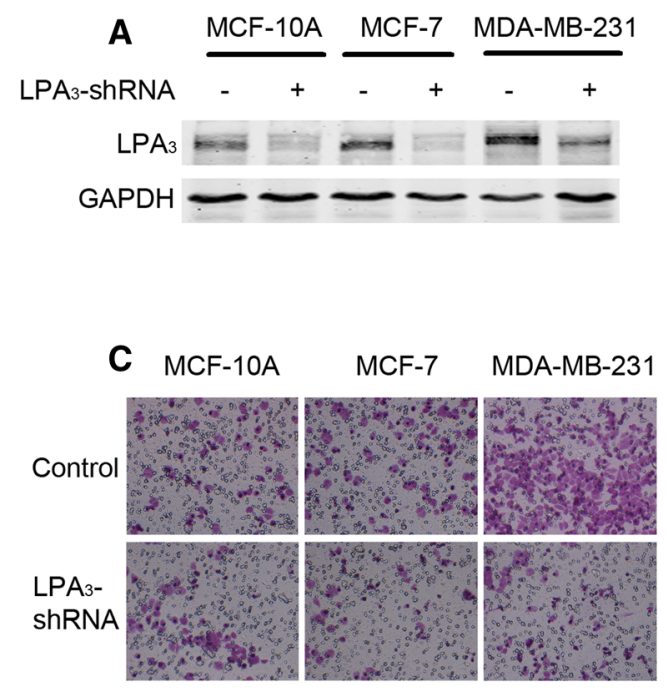

Fig. 5 Inactivated $\mathrm{LPA}_{3}$ by Ki16425 suppressed migration and invasion of TNBC cells. a MDA-MB-231 cells were treated with indicated concentrations of Ki16428 for $1 \mathrm{~h}$, and then cell viability was measured using the MTT assay. The results are presented as the mean \pm SD of fold increased to initiation obtained in 3 independent experiments. b, c Migration (b) and invasion (c) of MDA-MB-231 cells were inhibited by ki16425. MDAMB-231 cells were pretreated with indicated concentrations of ki16425 for $1 \mathrm{~h}$ and then transferred to collagen- or matrigel-coated transwell chambers for migration and invasion experiments, respectively. ${ }^{*} P<0.05$; $* * P<0.01 ; * * * P<0.001$
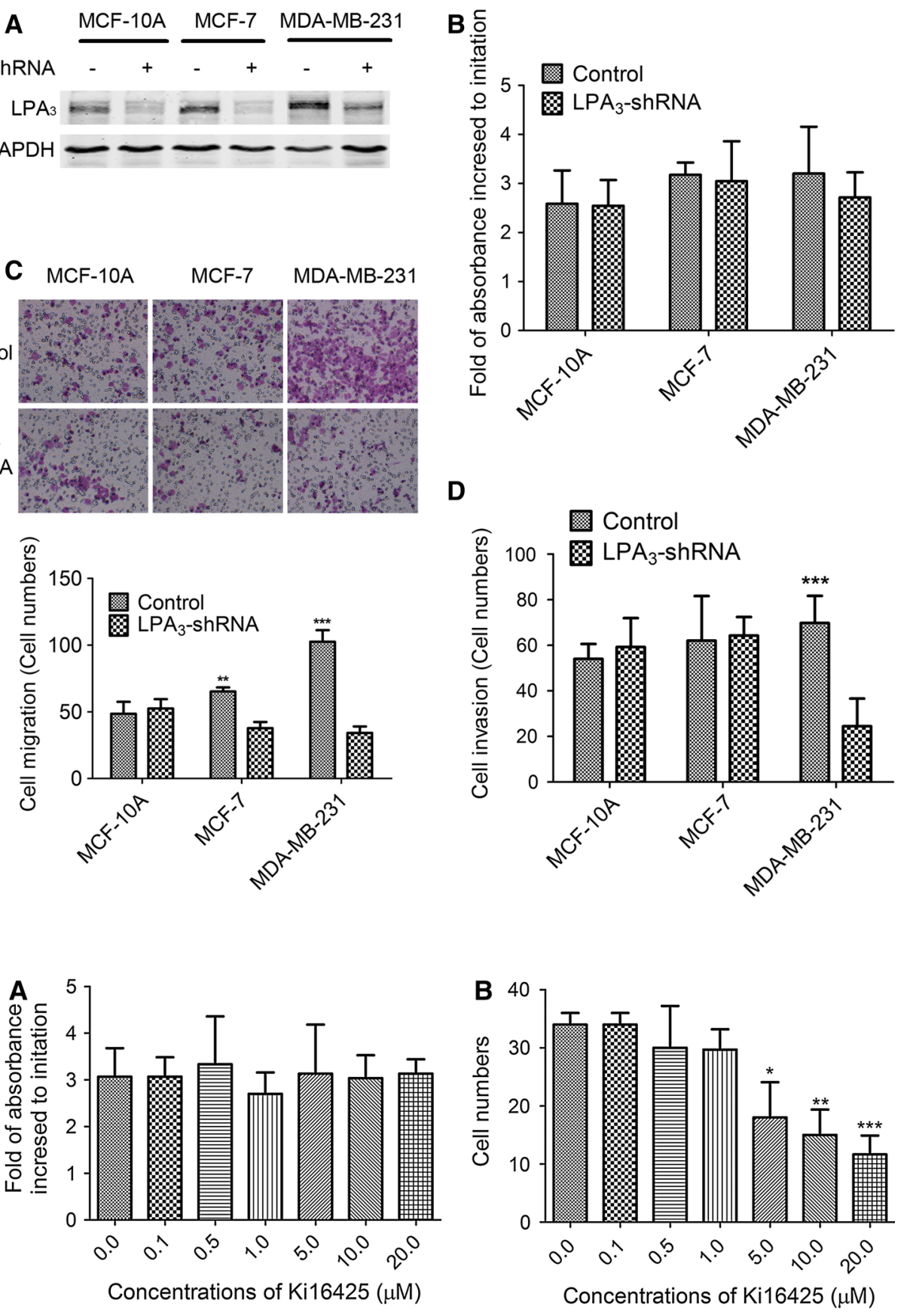
readily observed in breast cancer cells [19]. Manipulation of $\mathrm{LPA}_{1}$ level or function could alter the survival and metastatic ability of breast cancer cells both in vitro and in vivo $[11,12,14,20,21]$. However, in our present research, $\mathrm{LPA}_{1}$ expression levels did not differ significantly between normal and malignant breast tissues. This inconsistency may result from tumor heterogeneity. In breast cancer, $\mathrm{LPA}_{1}$ is expressed in many cancer cell lines, but at various levels. It is most likely that $\mathrm{LPA}_{1}$ higher expressed in more aggressive cell lines, such as MDA-MB231, and lower in less aggressive breast cancer cells, such as MCF-7 [10, 13, 20, 22, 23]. In accord with cell lines, $\mathrm{LPA}_{1}$ mRNA was significantly more abundant in advanced stages of breast cancer compared with noninvasive stage breast tumors [24]. Moreover, accumulating evidence indicates that the $\mathrm{LPA}_{1}$ contributes to the metastatic capability of breast cancers. Higher LPA $_{1}$ expression is significantly related to positive node and bone metastases [11, 12], which implies that $\mathrm{LPA}_{1}$ affects breast cancer progression. However, some clinical studies found no major expression pattern for the $\mathrm{LPA}_{1}$ between breast cancer patients and normal controls $[8,25]$, which suggests that $\mathrm{LPA}_{1}$ has no role in breast cancer initiation.

From an evolutionary perspective, tumors can be as genetically and epigenetically heterogeneous cell populations, although most human tumors are monoclonal outgrowths descending from single progenitor cells [26, 27]. As tumor progression, genetic and epigenetic alterations occur in progeny cells. However, changes in $\mathrm{LPA}_{1}$ expression as they affect breast cancer initiation and progression are barely understood and require additional exploration.

Although the expression and functions of $\mathrm{LPA}_{2}$ have been the subject of fewer studies, increased $\mathrm{LPA}_{2}$ expression has been reported in invasive breast carcinoma $[8,14]$. Transgenic mice that overexpress $\mathrm{LPA}_{2}$ showed higher incidence of mammary tumors with early onset than mice that overexpress $\mathrm{LPA}_{1}$, which implicates $\mathrm{LPA}_{2}$ in the initiation of breast cancer [14]. In vitro studies, $\mathrm{LPA}_{2}$ has been verified to regulate LPA-induced breast cancer cells proliferation and migration through Erk or RhoA pathway $[23,28]$. Recently, a literature also reported $\mathrm{LPA}_{2}$ involved in LPA-induced IL-6 and IL-8 expression, which promoted colony formation and cell survival of TNBCs [29]. Together with our findings that $\mathrm{LPA}_{2}$ is more highly expressed in breast cancer patients, these combined data validate $\mathrm{LPA}_{2}$ as a potential therapeutic target for drug development and evaluation in breast cancer.

As with $\mathrm{LPA}_{2}$, little is known about the expression patterns of $\mathrm{LPA}_{3}$ in breast cancers. Until recently, Nikolay et al. indicated that $\mathrm{LPA}_{3}$ was higher expressed in human breast cancers, and most interesting $\mathrm{LPA}_{3}$ overexpression was associated with absence of ER and PR [30], which suggests a function of $\mathrm{LPA}_{3}$ in $\mathrm{HR}^{-}$carcinomas. Our studies confirmed that $\mathrm{LPA}_{3}$ was overexpression in $\mathrm{ER}^{-} /$ $\mathrm{PR}^{-} / \mathrm{Her} 2^{-}$tumor cells and tissues when compared with normal breast epithelium and luminal-type cancers. Inhibition of $\mathrm{LPA}_{3}$ significantly decreased migration and invasion of TNBC cells but did not affect other immunotype breast cancers, reflecting on dominant metastatic roles of $\mathrm{LPA}_{3}$ in TNBCs.

Cancer metastasis is a complex biological event of multiple steps, one of which is epithelial to mesenchymal transition (EMT), a prelude to increased cellular motility and plasticity, which thereby enables cellular invasion [31, 32]. Initial evidence for a possible role of LPA and its receptors in EMT was derived from experiments in hepatocellular carcinoma and ovarian cancer by showing a proline-rich tyrosine kinase 2 (PYK2) or periostin (alias osteoblast-specific factor-2)-induced EMT, upon LPA treatment [33, 34]. In the breast cancer, Jahn et al. [35] demonstrated that $\mathrm{LPA}_{1}$ is upregulated in cells that underwent EMT and consequently led to an increased responsiveness to LPA after EMT. These results imply that the LPA receptors contribute to cell EMT. The roles of different LPA receptors in EMT clearly merit wider investigation.

As a receptor for LPA, $\mathrm{LPA}_{3}$ can promote cancer progression. However, the downstream pathways of $\mathrm{LPA}_{3}$ are rarely elucidated. Currently, evidences indicated that Yesassociated protein (YAP), a transcriptional factor of Hippo pathway, is a critical downstream mediatio of $\mathrm{LPA}_{3}$ in ovarian cancer [36]. Thus, we invested the expression correlation between $\mathrm{LPA}_{3}$ and YAP protein and found that tumors with overexpression of $\mathrm{LPA}_{3}$ exhibited week YAP staining (data not shown). As YAP functions as a breast tumor suppressor [37], $\mathrm{LPA}_{3}$-YAP pathway may involved in initiation and progression of breast cancers. Interesting, another study revealed that loss of YAP expression is associated with estrogen and PR negativity in breast carcinomas [38]. Besides, in transgenic mice model, the ER, $\mathrm{PR}$, and Her2 were significantly decreased in $\mathrm{LPA}_{3}$ overexpression mice when compared to wild-type mice [14]. All together, $\mathrm{LPA}_{3}, \mathrm{YAP}$, and hormonal receptors may interact in TNBC pathophysiology. However, the exact mechanism is unclear and requires further study.

Acknowledgments This work is supported by the National Natural Science Foundation of China (Grant No. 81171397).

Conflict of interest The authors declare no completing interests related to this work.

Open Access This article is distributed under the terms of the Creative Commons Attribution License which permits any use, distribution, and reproduction in any medium, provided the original author(s) and the source are credited. 


\section{References}

1. Jemal A, Bray F, Center MM, Ferlay J, Ward E, Forman D. Global cancer statistics. CA Cancer J Clin. 2011;61(2):69-90. doi:10.3322/caac.20107.

2. Poage GM, Hartman ZC, Brown PH. Revealing targeted therapeutic opportunities in triple-negative breast cancers: a new strategy. Cell Cycle. 2013;12(17):2705-6. doi:10.4161/cc.25871.

3. Lehmann BD, Bauer JA, Chen X, Sanders ME, Chakravarthy AB, Shyr $\mathrm{Y}$, et al. Identification of human triple-negative breast cancer subtypes and preclinical models for selection of targeted therapies. J Clin Investig. 2011;121(7):2750-67. doi:10.1172/ JCI45014.

4. Speers C, Tsimelzon A, Sexton K, Herrick AM, Gutierrez C, Culhane A, et al. Identification of novel kinase targets for the treatment of estrogen receptor-negative breast cancer. Clin Cancer Res. 2009;15(20):6327-40. doi:10.1158/1078-0432.CCR-091107.

5. Mohamed A, Krajewski K, Cakar B, Ma CX. Targeted therapy for breast cancer. Am J Pathol. 2013;183(4):1096-112. doi:10. 1016/j.ajpath.2013.07.005.

6. Gotoh M, Fujiwara Y, Yue J, Liu J, Lee S, Fells J, et al. Controlling cancer through the autotaxin-lysophosphatidic acid receptor axis. Biochem Soc Trans. 2012;40(1):31-6. doi:10.1042/ BST20110608.

7. Willier S, Butt E, Grunewald TG. Lysophosphatidic acid (LPA) signalling in cell migration and cancer invasion: a focussed review and analysis of LPA receptor gene expression on the basis of more than 1700 cancer microarrays. Biol Cell. 2013;105(8):317-33. doi:10.1111/boc.201300011.

8. Kitayama J, Shida D, Sako A, Ishikawa M, Hama K, Aoki J, et al. Over-expression of lysophosphatidic acid receptor-2 in human invasive ductal carcinoma. Breast Cancer Res. 2004;6(6):R640-6. doi:10.1186/bcr935.

9. Wu J, Mukherjee A, Lebman DA, Fang X. Lysophosphatidic acid-induced p21Waf1 expression mediates the cytostatic response of breast and ovarian cancer cells to TGFbeta. Mol Cancer Res. 2011;9(11):1562-70. doi:10.1158/1541-7786.MCR11-0340.

10. Hama K, Aoki J, Fukaya M, Kishi Y, Sakai T, Suzuki R, et al. Lysophosphatidic acid and autotaxin stimulate cell motility of neoplastic and non-neoplastic cells through LPA1. J Biol Chem. 2004;279(17):17634-9. doi:10.1074/jbc.M313927200.

11. David M, Ribeiro J, Descotes F, Serre CM, Barbier M, Murone $\mathrm{M}$, et al. Targeting lysophosphatidic acid receptor type 1 with Debio 0719 inhibits spontaneous metastasis dissemination of breast cancer cells independently of cell proliferation and angiogenesis. Int J Oncol. 2012;40(4):1133-41. doi:10.3892/ijo.2011. 1309.

12. Boucharaba A, Serre CM, Guglielmi J, Bordet JC, Clezardin P, Peyruchaud O. The type 1 lysophosphatidic acid receptor is a target for therapy in bone metastases. Proc Natl Acad Sci USA. 2006;103(25):9643-8. doi:10.1073/pnas.0600979103.

13. Samadi N, Bekele RT, Goping IS, Schang LM, Brindley DN. Lysophosphatidate induces chemo-resistance by releasing breast cancer cells from taxol-induced mitotic arrest. PLoS One. 2011;6(5):e20608. doi:10.1371/journal.pone.0020608.

14. Liu S, Umezu-Goto M, Murph M, Lu Y, Liu W, Zhang F, et al. Expression of autotaxin and lysophosphatidic acid receptors increases mammary tumorigenesis, invasion, and metastases. Cancer Cell. 2009;15(6):539-50. doi:10.1016/j.ccr.2009.03.027.

15. Popnikolov NK, Dalwadi BH, Thomas JD, Johannes GJ, Imagawa WT. Association of autotaxin and lysophosphatidic acid receptor 3 with aggressiveness of human breast carcinoma. Tumour Biol. 2012;33(6):2237-43. doi:10.1007/s13277-012-0485-1.
16. Sengupta S, Xiao YJ, Xu Y. A novel laminin-induced LPA autocrine loop in the migration of ovarian cancer cells. FASEB J. 2003;17(11):1570-2. doi:10.1096/fj.02-1145fje.

17. Sorlie T, Perou CM, Tibshirani R, Aas T, Geisler S, Johnsen H, et al. Gene expression patterns of breast carcinomas distinguish tumor subclasses with clinical implications. Proc Natl Acad Sci USA. 2001;98(19):10869-74. doi:10.1073/pnas.191367098.

18. Panupinthu N, Lee HY, Mills GB. Lysophosphatidic acid production and action: critical new players in breast cancer initiation and progression. Br J Cancer. 2010;102(6):941-6. doi:10.1038/sj. bjc. 6605588 .

19. Witt AE, Hines LM, Collins NL, Hu YH, Gunawardane RN, Moreira D, et al. Functional proteomics approach to investigate the biological activities of cDNAs implicated in breast cancer. J Proteome Res. 2006;5(3):599-610. doi:10.1021/Pr050395i.

20. Goetzl EJ, Dolezalova H, Kong Y, Zeng L. Dual mechanisms for lysophospholipid induction of proliferation of human breast carcinoma cells. Cancer Res. 1999;59(18):4732-7.

21. Hama K, Aoki J, Fukaya M, Kishi Y, Sakai T, Suzuki R. Lysophosphatidic acid and autotaxin stimulate cell motility of neoplastic and non-neoplastic cells through LPA(1). J Biol Chem. 2004;279(17):17634-9. doi:10.1074/jbc.M313927200.

22. Swamydas M, Nguyen D, Allen LD, Eddy J, Dreau D. Progranulin stimulated by LPA promotes the migration of aggressive breast cancer cells. Cell Commun Adhes. 2011;18(6):119-30. doi: $10.3109 / 15419061.2011 .641042$.

23. Chen M, Towers LN, O'Connor KL. LPA2 (EDG4) mediates Rho-dependent chemotaxis with lower efficacy than LPA1 (EDG2) in breast carcinoma cells. Am J Physiol Cell Physiol. 2007;292(5):C1927-33. doi:10.1152/ajpcell.00400.2006.

24. Li TT, Alemayehu M, Aziziyeh AI, Pape C, Pampillo M, Postovit LM, et al. Beta-arrestin/Ral signaling regulates lysophosphatidic acid-mediated migration and invasion of human breast tumor cells. Mol Cancer Res. 2009;7(7):1064-77. doi:10.1158/15417786.MCR-08-0578.

25. Murph MM, Nguyen GH, Radhakrishna H, Mills GB. Sharpening the edges of understanding the structure/function of the LPA1 receptor: expression in cancer and mechanisms of regulation. BBA Mol Cell Biol Lipids. 2008;1781(9):547-57. doi:10.1016/j. bbalip.2008.04.007.

26. Grunewald TG, Herbst SM, Heinze J, Burdach S. Understanding tumor heterogeneity as functional compartments-superorganisms revisited. J Transl Med. 2011;9:79. doi:10.1186/1479-58769-79.

27. Merlo LM, Pepper JW, Reid BJ, Maley CC. Cancer as an evolutionary and ecological process. Nat Rev Cancer. 2006;6(12):924-35. doi:10.1038/nrc2013.

28. Wu JH, Mukherjee A, Lebman DA, Fang XJ. Lysophosphatidic acid-induced p21(Wafl) expression mediates the cytostatic response of breast and ovarian cancer cells to TGF beta. Mol Cancer Res. 2011;9(11):1562-70. doi:10.1158/1541-7786.Mcr11-0340.

29. Hartman ZC, Poage GM, den Hollander P, Tsimelzon A, Hill J, Panupinthu N, et al. Growth of triple-negative breast cancer cells relies upon coordinate autocrine expression of the proinflammatory cytokines IL-6 and IL-8. Cancer Res. 2013;73(11):3470-80. doi:10.1158/0008-5472.Can-12-4524-T.

30. Popnikolov NK, Dalwadi BH, Thomas JD, Johannes GJ, Imagawa WT. Association of autotaxin and lysophosphatidic acid receptor 3 with aggressiveness of human breast carcinoma. Tumor Biol. 2012;33(6):2237-43. doi:10.1007/s13277-012-0485-1.

31. Bullock MD, Sayan AE, Packham GK, Mirnezami AH. MicroRNAs: critical regulators of epithelial to mesenchymal (EMT) and mesenchymal to epithelial transition (MET) in cancer progression. Biol Cell. 2012;104(1):3-12. doi:10.1111/boc. 201100115. 
32. De Craene B, Berx G. Regulatory networks defining EMT during cancer initiation and progression. Nat Rev Cancer. 2013;13(2):97-110. doi:10.1038/nrc3447.

33. Choi KU, Yun JS, Lee IH, Heo SC, Shin SH, Jeon ES, et al. Lysophosphatidic acid-induced expression of periostin in stromal cells: prognostic relevance of periostin expression in epithelial ovarian cancer. Int J Cancer. 2011;128(2):332-42. doi:10.1002/ ijc. 25341

34. Sun CK, Ng KT, Lim ZX, Cheng Q, Lo CM, Poon RT, et al. Proline-rich tyrosine kinase 2 (Pyk2) promotes cell motility of hepatocellular carcinoma through induction of epithelial to mesenchymal transition. PLoS One. 2011;6(4):e18878. doi:10. 1371/journal.pone.0018878.

35. Jahn SC, Law ME, Corsino PE, Parker NN, Pham K, Davis BJ, et al. An in vivo model of epithelial to mesenchymal transition reveals a mitogenic switch. Cancer Lett. 2012;326(2):183-90. doi:10.1016/j.canlet.2012.08.013.

36. Cai $\mathrm{H}, \mathrm{Xu} \mathrm{Y}$. The role of LPA and YAP signaling in long-term migration of human ovarian cancer cells. Cell Commun Signal. 2013;11. doi:10.1186/1478-811x-11-31.

37. Yuan M, Tomlinson V, Lara R, Holliday D, Chelala C, Harada T, et al. Yes-associated protein (YAP) functions as a tumor suppressor in breast. Cell Death Differ. 2008;15(11):1752-9. doi:10. 1038/cdd.2008.108.

38. Tufail R, Jorda M, Zhao W, Reis I, Nawaz Z. Loss of Yesassociated protein (YAP) expression is associated with estrogen and progesterone receptors negativity in invasive breast carcinomas. Breast Cancer Res Treat. 2012;131(3):743-50. doi:10. 1007/s10549-011-1435-0. 